\title{
Supporting Info: In Situ Probing Potassium-ion Intercalation-induced Amorphization in Crystalline Iron Phosphate Cathode Materials
}

\author{
Bertan Özdogru, Younghwan Cha, Bharat Gwalani, Vijayakumar Murugesan, \\ Min-Kyu Song and Ömer Özgür Çapraz*
}

Bertan Özdogru, Prof. Ömer Özgür Çapraz*

The School of Chemical Engineering

Oklahoma State University

Stillwater, OK 74078

E-mail: ocapraz@okstate.edu

Dr. Younghwan Cha, Prof. Min-Kyu Song,

School of Mechanical and Materials Engineering

Washington State University

Pullman, WA 99164

Dr. Bharat Gwalani, Dr. Vijayakumar Murugesan

Joint Center for Energy Storage Research

Pacific Northwest National Laboratory

902 Battelle Blvd, Richland, WA 99354 


\section{EXPERIMENTAL}

Sample Preparation: Composite electrodes were prepared by mixing active material with binder and conductive carbon in 8:1:1 mass ratio. Initially, sodium carboxymethyl cellulose (binder, $\mathrm{CMC}$, average $\mathrm{MW} \sim 700,000$, Aldrich) was dissolved in ultra-pure water with a 1:40 mass ratio. Then, the pristine lithium iron phosphate (active material, $\mathrm{LiFePO}_{4}$, LFP, Hanwha Chemical) and Super P (conductive additive, carbon black, $>99 \%$, metal basis, Alfa Aesar) were added to the binder solution. Scanning electron microscopy (SEM) images of the electrode particle are shown in the Supp. Fig 1. The average particle size of LFP particles was measured by SEM as $219 \pm 78$ $\mathrm{nm}$. Particle size ranges from $100 \mathrm{~nm}$ to $400 \mathrm{~nm}$. The slurry was mixed for 30 minutes with Thinky centrifugal mixer at 2000 RPM mixing speed. Composite electrodes were cast onto the copper foil (9 $\mu \mathrm{m}$ thick, $>99.99 \%$, MTI) with a doctor blade to control the slurry thickness. The slurry was then dried under ambient conditions for $16 \mathrm{~h}$. Dried electrodes were carefully peeled off to create the freestanding electrode for strain measurements.

Electrolyte solution was prepared by mixing ethylene carbonate (EC, anhydrous, 99\%, Acros Organics) and dimethyl carbonate (DMC, anhydrous, $>99 \%$, Aldrich) in 1:1 volume ratio inside the glovebox under an argon atmosphere. Oxygen and water content inside the glovebox kept below $1 \mathrm{ppm}$ all the time. $1 \mathrm{M}$ lithium perchlorate $\left(\mathrm{LiClO}_{4}\right.$, battery grade, dry, $99.99 \%<$ Aldrich) or $0.5 \mathrm{M} \mathrm{KPF}_{6}$ ( $99 \%$ min, Alfa Aesar) was added to the EC/DMC solution for $\mathrm{Li}$, Na or $\mathrm{K}$ intercalation, respectively.

Li foils (99.9\% metal basis, Alfa Aesar) were used as purchased without proceeding any further treatment and were kept inside the argon-filled glove box. Potassium chunks (98\%, Acros Organics), immersed in mineral oil, were cleaned with hexane inside the glovebox. Cleaned $\mathrm{K}$ chunks were stored in EC:DMC solution in a 1:1 volume ratio for future use. Prior to custom cell assembly, $\mathrm{K}$ chunks were removed from the solvent solution, dried with a filter paper, and cut into pieces with a stainless-steel scalpel. The cut piece was placed inside a polyethylene bag and rolled into the shape of a foil using a rolling pin. Flattened $\mathrm{K}$ foil was then placed into the battery cell as a counter electrode. 


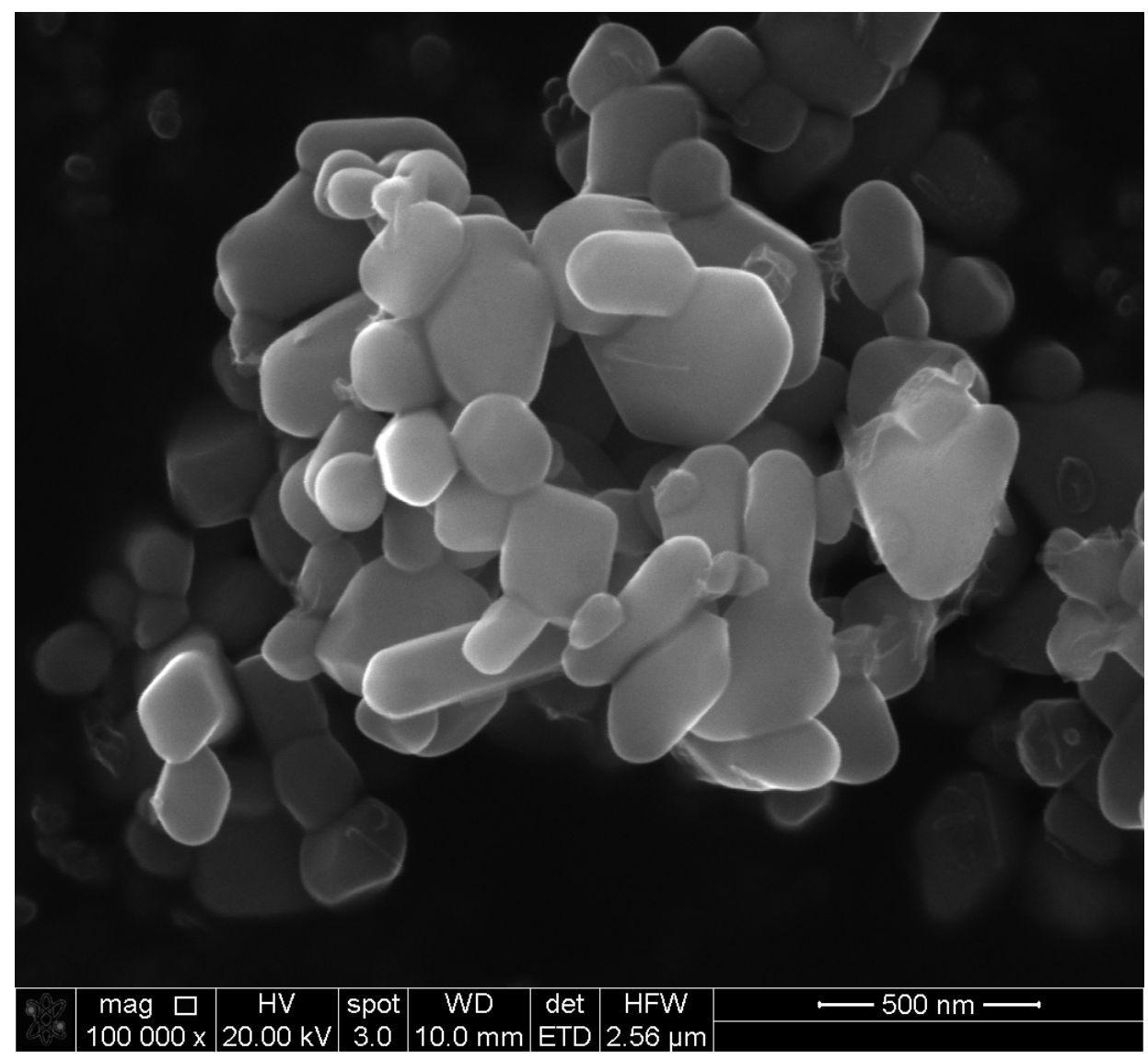

Supp. Figure 1: SEM image of the pristine electrode particles

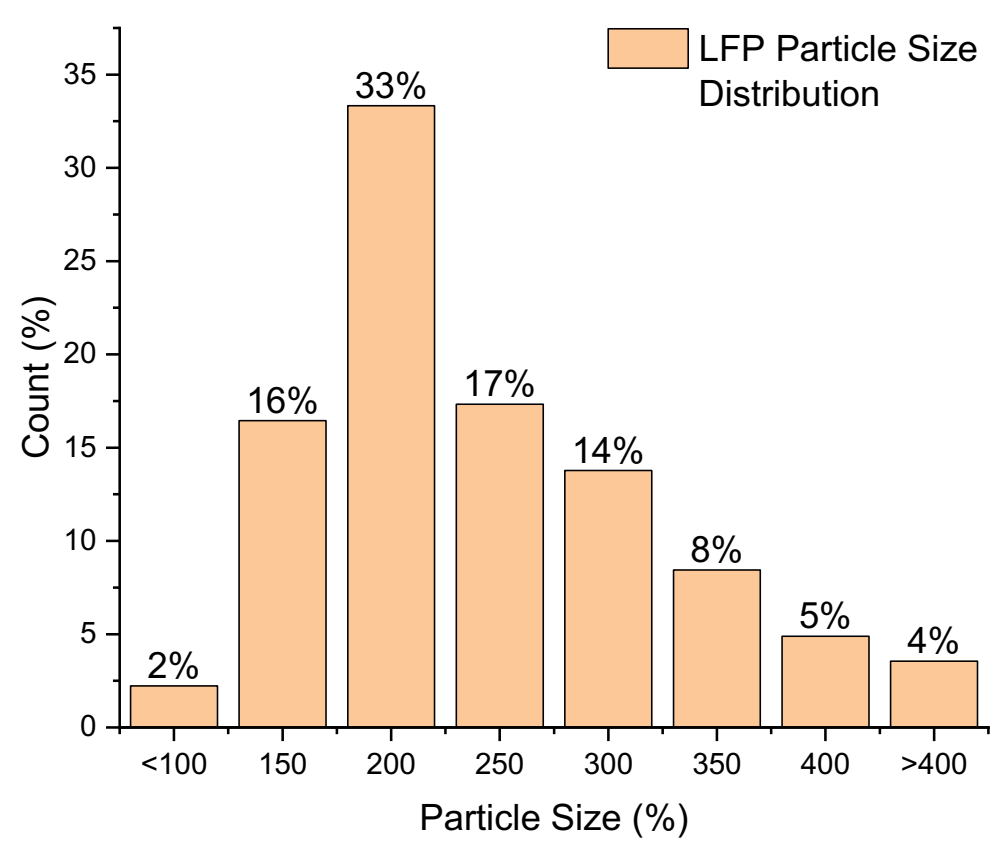

Supp. Figure 2: Particle size distribution of Lithium Iron Phosphate cathode particles from 225 particles. 
Battery Cycling: Iron phosphate (FP) composite electrode was formed by electrochemical displacement technique using a pristine LFP composite electrode ${ }^{1,2}$. The pristine LFP electrode was electrochemically delithiated via galvanostatic cycle against $\mathrm{K}$ counter electrode at a rate of $\mathrm{C} / 25$. For potassium intercalation, $\mathrm{FP}$ composite electrodes were cycled against $\mathrm{K}$ counter electrode in 0.5 M KPF 6 in 1:1 (v:v) EC:DMC electrolyte between 1.5-4.3 V. The electrodes were cycled via galvanostatic cycle at $\mathrm{C} / 25$ rate.

In-operando XRD Measurements: X-ray diffractometer (SmartLab, Rigaku) equipped with an inoperando battery cell was employed for the XRD analysis. LFP slurry was drop-casted directly on Beryllium window and dried overnight in ambient condition. The loading of LFP was measured to be approximately $2 \mathrm{mg} / \mathrm{cm}^{2}$. With the prepared LFP cathode, the in-operando battery cell was assembled in Ar filled glove box where moisture and oxygen level were controlled to be below $0.5 \mathrm{ppm}$. K metal electrode and electrolyte were prepared in a same manner described above. Two glass fiber separators were used between the K metal anode and LFP cathode. The XRD patterns were continuously collected over the electrochemical displacement process at $\mathrm{C} / 10$ up to $4.3 \mathrm{~V}$ and subsequent discharge/change cycling process at $\mathrm{C} / 25$ between $1.5 \sim 4.3 \mathrm{~V}(1 \mathrm{C}=141 \mathrm{mAh} / \mathrm{g}$ of active

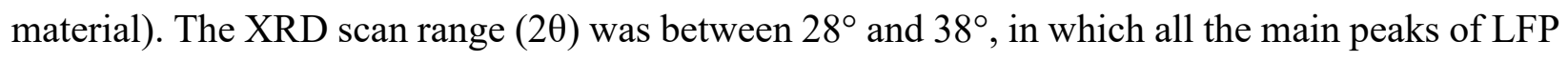
and FP can be observed. Every scan took approximately 20 minutes. The step width used in the XRD measurement was $0.01^{\circ}$.

High Resolution Transmission Electron Microscopy Measurements: The sample preparation for high-resolution transmission electron microscopy (HRTEM) was performed using a FEI Helios Hydra UX Focused Ion Beam-Scanning Electron Microscope (SEM) using Xe ions, outfitted with an Oxford Instruments X-ray Energy Dispersive Spectroscopy (EDS) system for compositional analysis. An aberration-corrected TEM (Titan 80-300 Scanning TEM/ STEM from Thermo Fisher Scientific, USA) equipped with a high-angle annular dark-field (HAADF) detector and an EDS system was employed at $300 \mathrm{kV}$ for bright-field (BF), HRTEM, high angle annular dark field (HAADF) STEM, selected area electron diffraction (SAED), and composition analysis. 
Strain Measurements: A detailed description of the custom battery cell was described in our previous publication ${ }^{3,4}$. Supp. Figure 1 and 2 shows the experimental setup and custom battery cell for the in-situ strain measurement system. The main body of the custom battery cell and the electrode holders were made from polychlorotrifluoroethylene (PCTFE, Plastics International). For optical access, a quartz window $\left(99.995 \% \mathrm{SiO}_{2}, 1 / 16\right.$ in thick, 2 in diameter, McMaster-Carr) was placed on the top of the custom cell and sealed with Viton O-rings (Grainger).

Analysis of the strain generation was carried out by taking images of the freestanding electrode throughout the electrochemical cycling periods. Images were captured with Grasshopper3 5.0 MP camera (Sony IMX250, resolution, 2448w x 2048h pixel) with 12.0X adjustable zoom lens (NAVITAR) for an effective resolution of $0.873 \mu \mathrm{m} /$ pixel when using $4.0 \mathrm{X}$ setting on the adjustable zoom lens. A LED light source (Amazon) was used for illumination of the electrode surface. Images were captured using a lab-made LabView program every 2 minutes during galvanostatic cycling at $\mathrm{C} / 25$. Captured images during electrochemical cycling were analyzed by Vic-2D Digital Image Correlation software. The software tracks the changes in the speckle pattern positions on the electrode surface, taking the initial image as reference, to calculate the strain evolution during the experiment. The natural speckle pattern of the LFP composite electrode was used to calculate strain generation on the electrode using Digital Image Correlation. Full-field strain measurements were performed on an area of interest of $750 \mu \mathrm{m} \times 500 \mu \mathrm{m}$. Strains were synchronized with the electrochemical response of the electrodes (current and voltage) using a lab-made MATLAB program. An example calculation of in situ strains on the composite electrode is shown in the Supp. Fig 2. Counter plots shows the homogeneous average of the individual particle response. For each image captured during cycling, $\varepsilon_{x x}$ is averaged over the area of the region of interest. 
A)

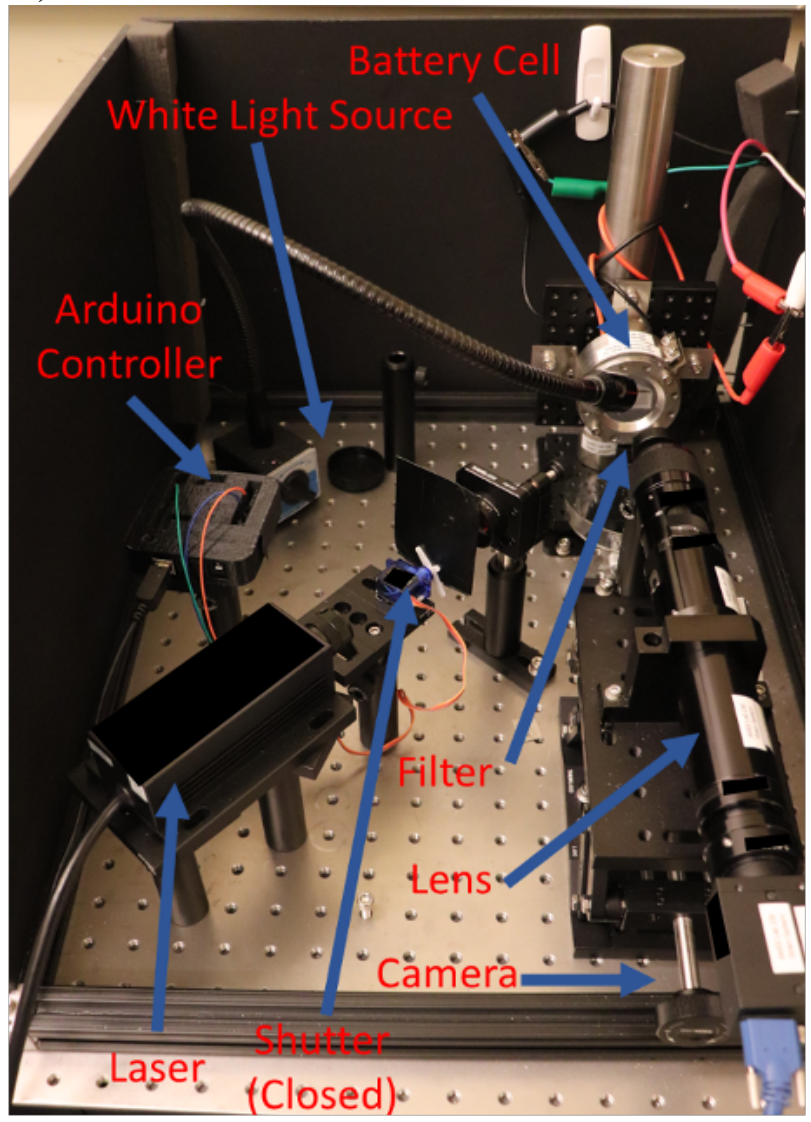

B)

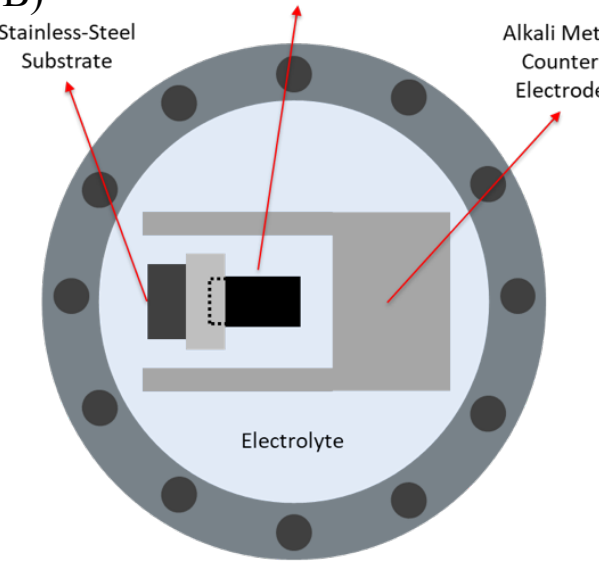

C)

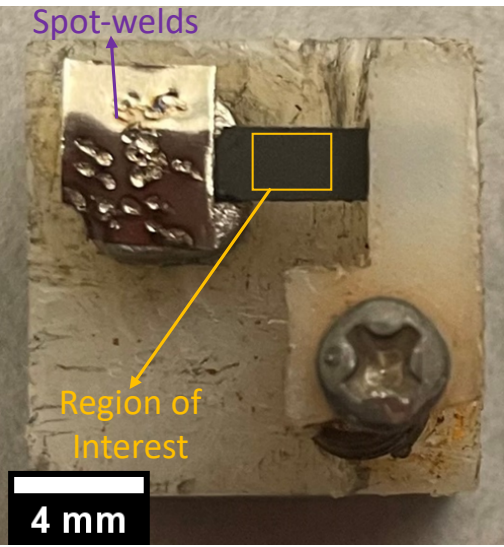

Supp. Figure 3: A) In-situ strain measurement setup and its components. B) Schematic view of the custom cell. C) Magnified view of a composite electrode spot welded on the stainless steel. The orange marked area on the Fig is the region of interest for the strain analysis. 

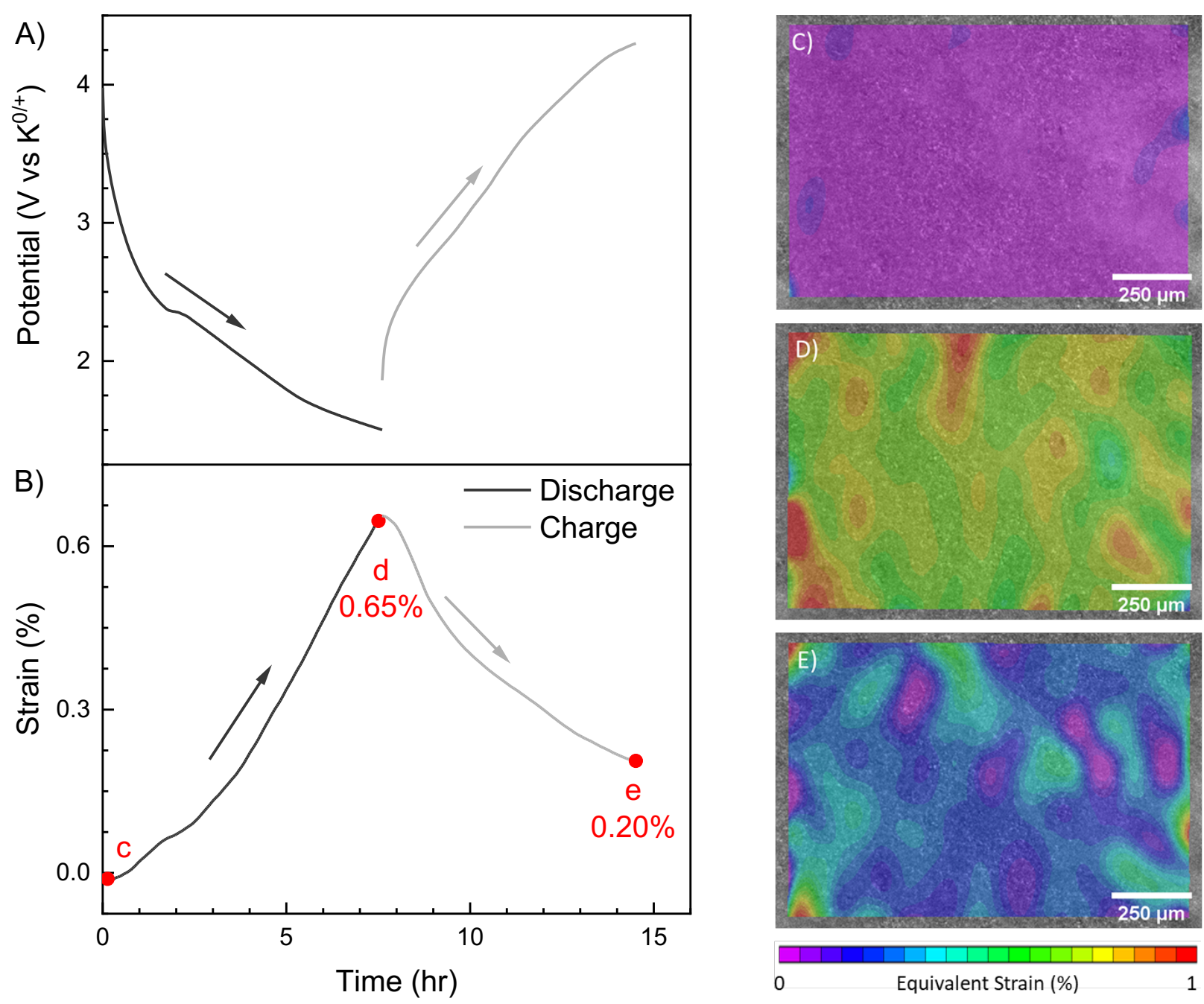

Supp. Figure 4: (A) Voltage and (B) strain evolution in iron phosphate electrode for potassium intercalation at $\mathrm{C} / 25$ rate with $0.5 \mathrm{M} \mathrm{KPF}_{6}$ in $\mathrm{EC} / \mathrm{DMC}$ electrolyte during first cycle. DIC contour plots show the equivalent strain generation for (C) at the beginning of discharge, (D) at the end of $1^{\text {st }}$ discharge and (E) at the end of first charge. 
Supp. Table 1: Interplanar spacing in iron phosphate electrode structure upon potassium intercalation during first discharge cycle.

\begin{tabular}{cccccc}
\hline (hkl) & & $\mathbf{( 3 1 1 )}$ & $\mathbf{( 1 2 1 )}$ & $\mathbf{( 2 1 1 )}$ & $\mathbf{( 0 2 0 )}$ \\
\hline Before & $2 \theta$ & 36.47 & 37.2 & 30.06 & 30.63 \\
\cline { 2 - 6 } Discharge & $\mathrm{d}(\mathrm{nm})$ & 0.246 & 0.242 & 0.297 & 0.292 \\
\hline $\begin{array}{c}\text { After } \\
\text { Discharge }\end{array}$ & $2 \theta$ & 36.4 & 37.1 & 29.94 & 30.53 \\
\cline { 2 - 6 } Change & $\mathrm{d}(\mathrm{nm})$ & 0.247 & 0.242 & 0.298 & 0.293 \\
\hline
\end{tabular}




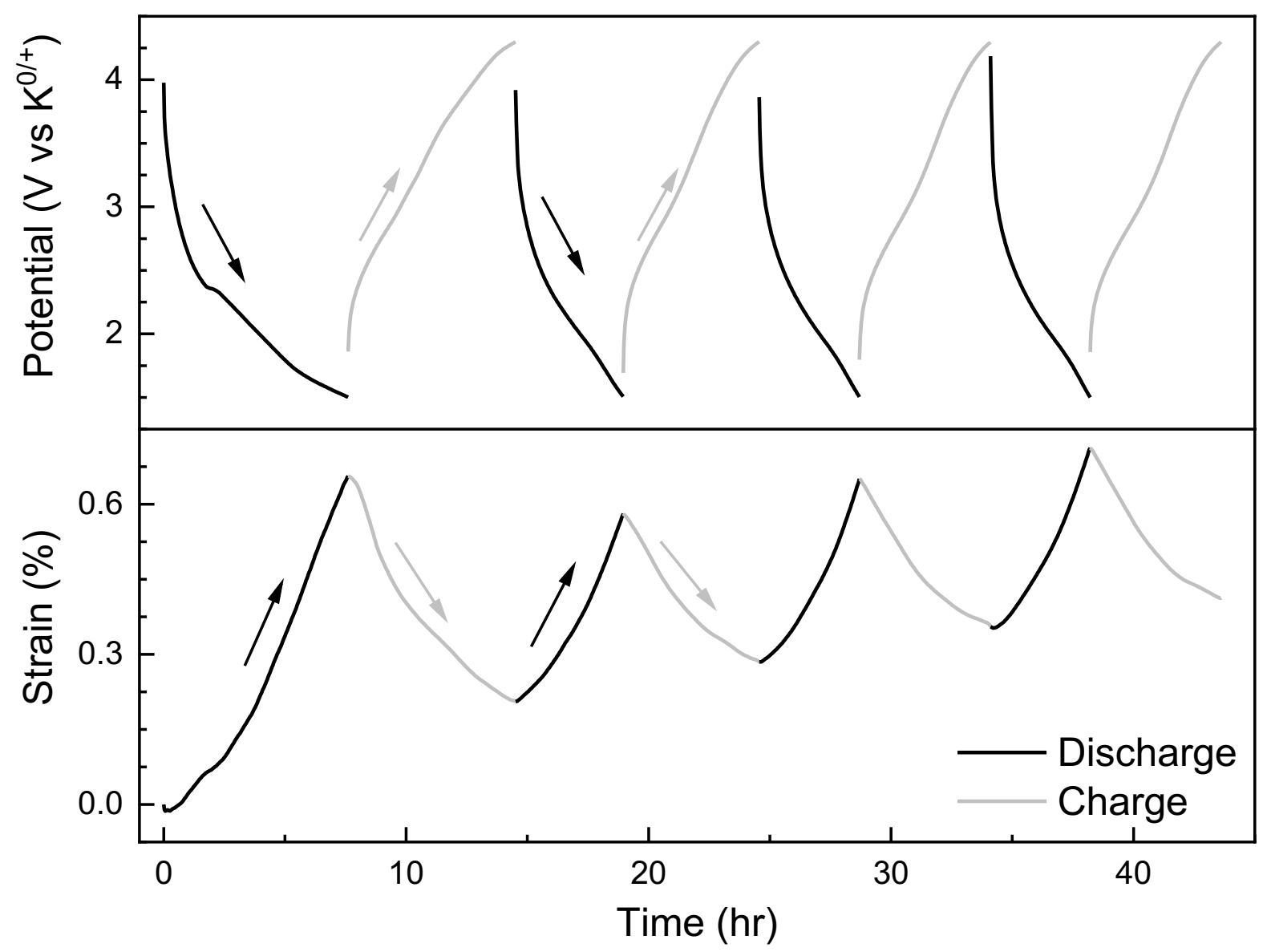

Supp. Figure 5: Voltage and strain evolution in iron phosphate electrode during potassium intercalation at $\mathrm{C} / 25$ rate in $0.5 \mathrm{M} \mathrm{KPF}_{6}$ in $\mathrm{EC} / \mathrm{DMC}$ electrolyte 

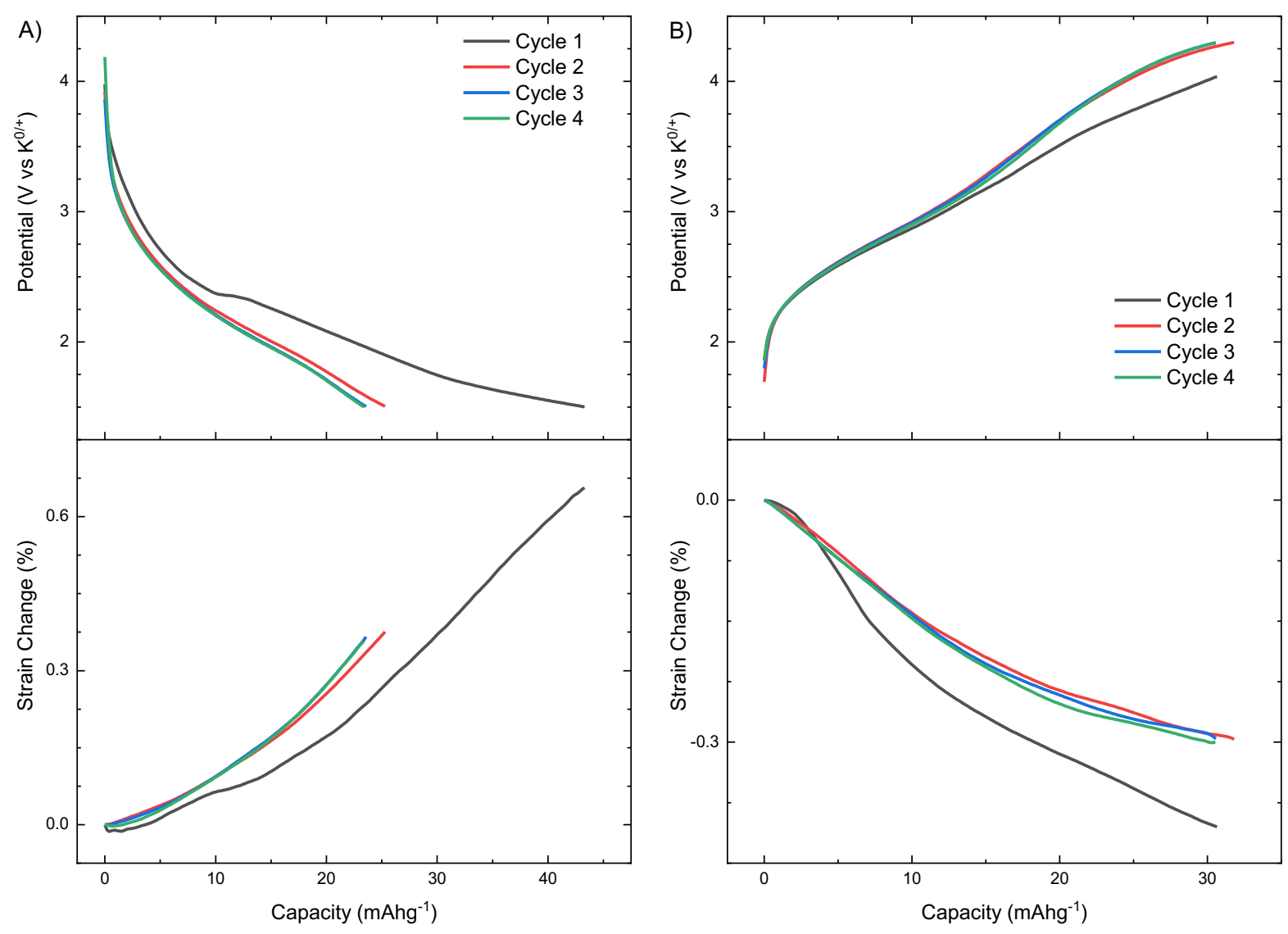

Supp. Figure 6: Change in strain versus (A) discharge and (B) charge capacity in iron phosphate during potassium intercalation at $\mathrm{C} / 25$ rate in $0.5 \mathrm{M} \mathrm{KPF}_{6}$ in $\mathrm{EC} / \mathrm{DMC}$ electrolyte. Strain values are set to zero at the beginning of each charge and discharge cycles for better comparison. 

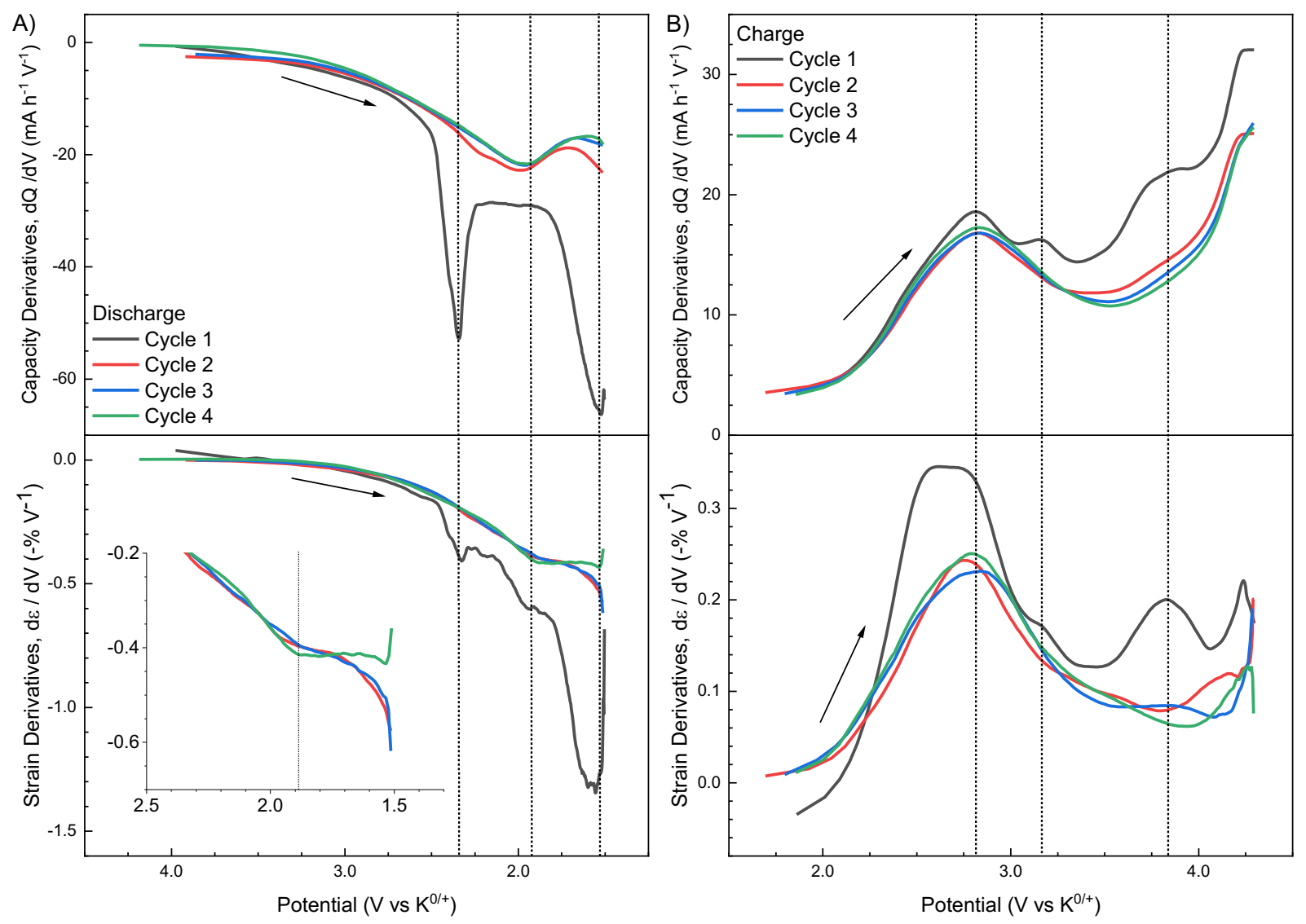

Supp. Figure 7: Capacity and strain derivatives in iron phosphate electrode during potassium intercalation for (A) discharge and (B) charge at $\mathrm{C} / 25$ rate in $0.5 \mathrm{M} \mathrm{KPF}_{6}$ in $\mathrm{EC} / \mathrm{DMC}$ electrolyte 
Control Experiment:. First, the LFP electrode is delithiated against Li counter metal in LiClO4 in EC:DMC electrolyte in order to form iron phosphate. Then, Li counter metal is removed. Delithiated composite electrode was rinsed and the custom cell is washed with 1:1 EC:DMC electrolyte inside the glovebox. Then, battery cell was reconstructed with $\mathrm{K}$ metal counter electrode and filled with $0.5 \mathrm{M} \mathrm{KPF}_{6}$ in 1:1 EC:DMC electrolyte. The iron phosphate electrode is charged / discharged against K metal while monitoring in situ strains as shown in Supp. Fig XXX below;

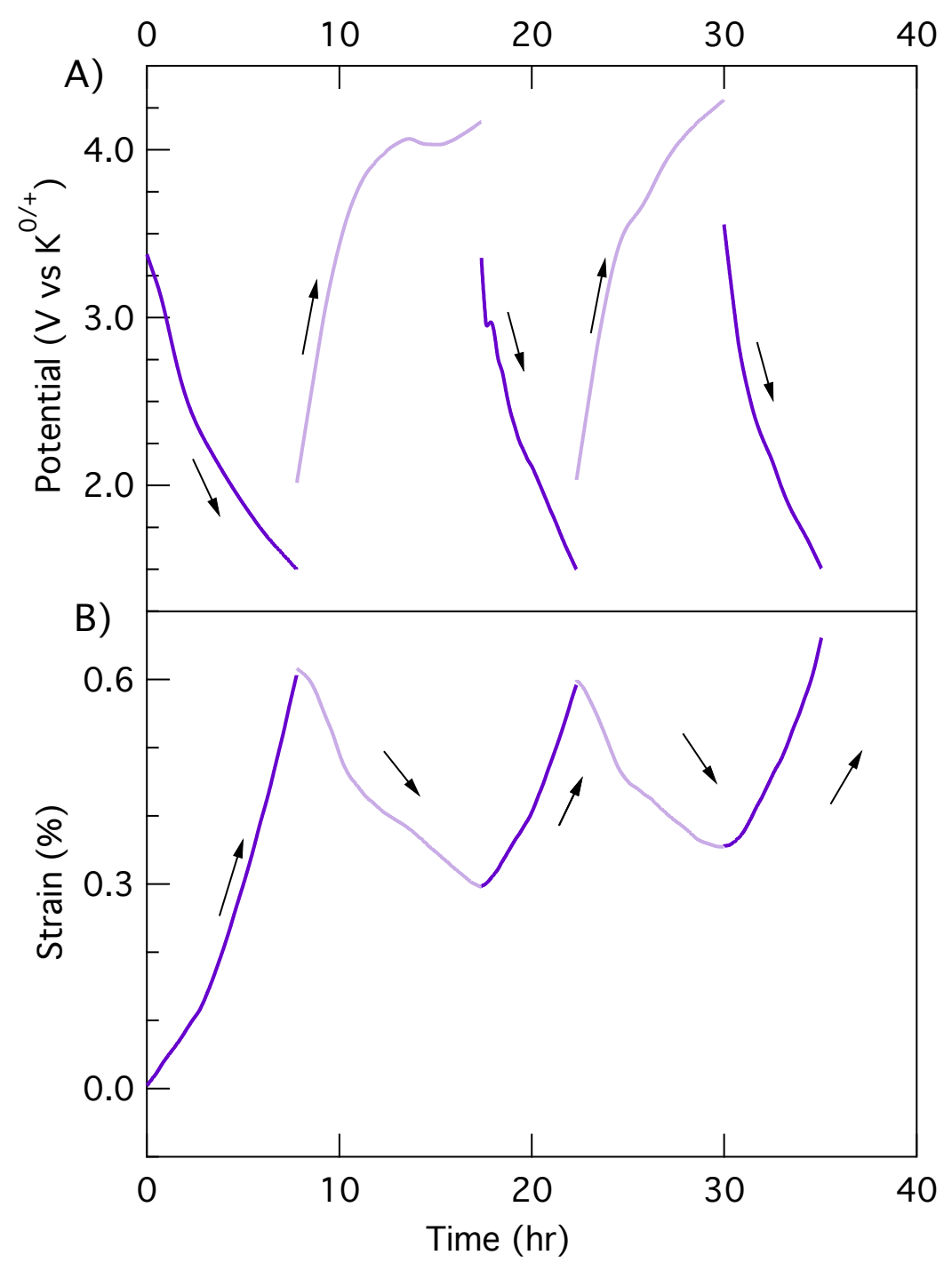

Supp. Figure 8: Voltage and strain evolution with time for the interrupted experiment against $\mathrm{K}$ counter electrode using 0.5 M KPF6 in 1:1 EC:DMC electrolyte during cycling. 

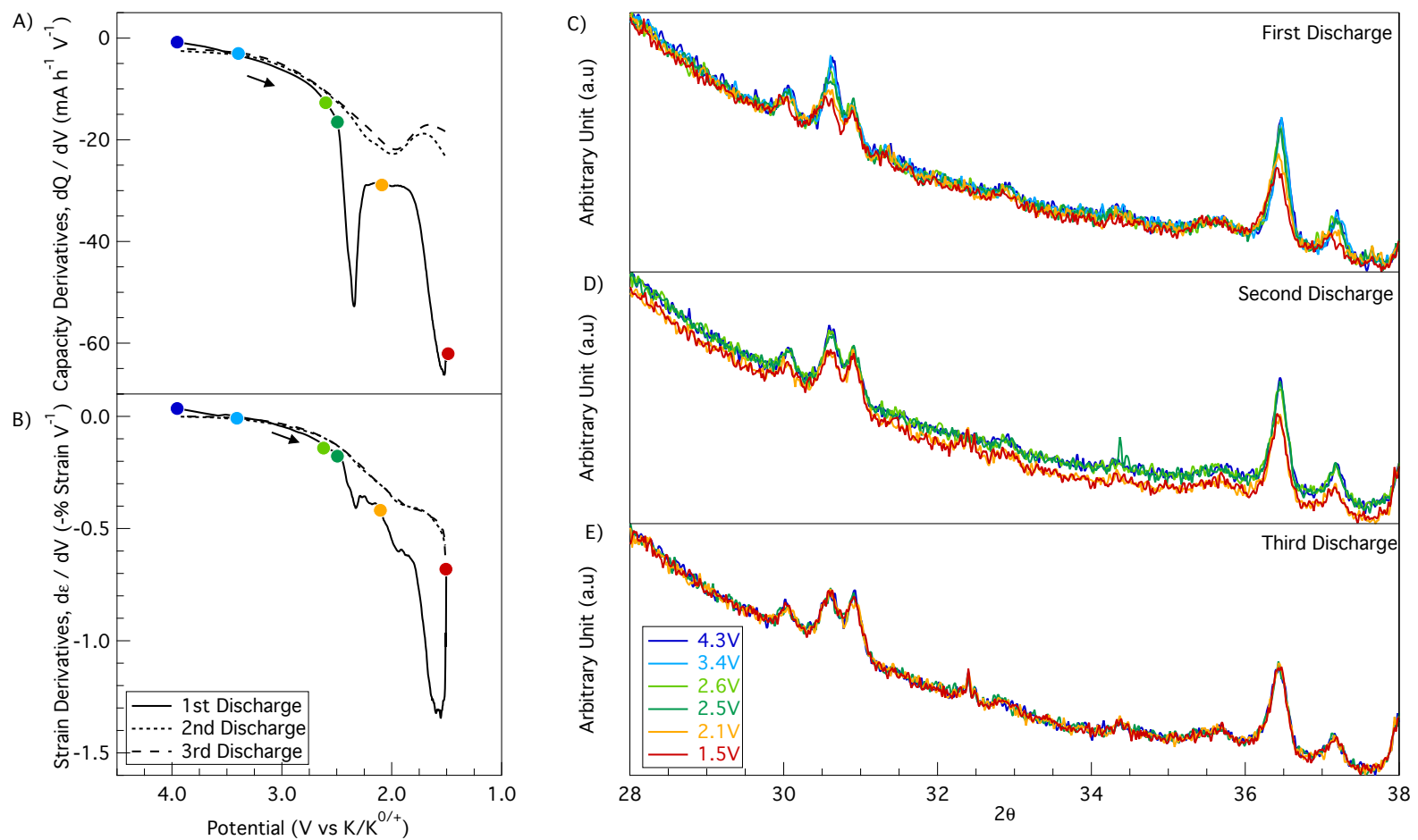

Supp. Fig 9: The figure is same as the Fig 4 in the manuscript. Only, the XRD patterns are plotted on top of each other in this figure. Structural, physical and electrochemical response of the iron phosphate during first three discharge cycles A) capacity and B) strain derivatives with respect to voltage. C-E) Corresponding XRD patterns at selected potentials colored as shown in the figure. 


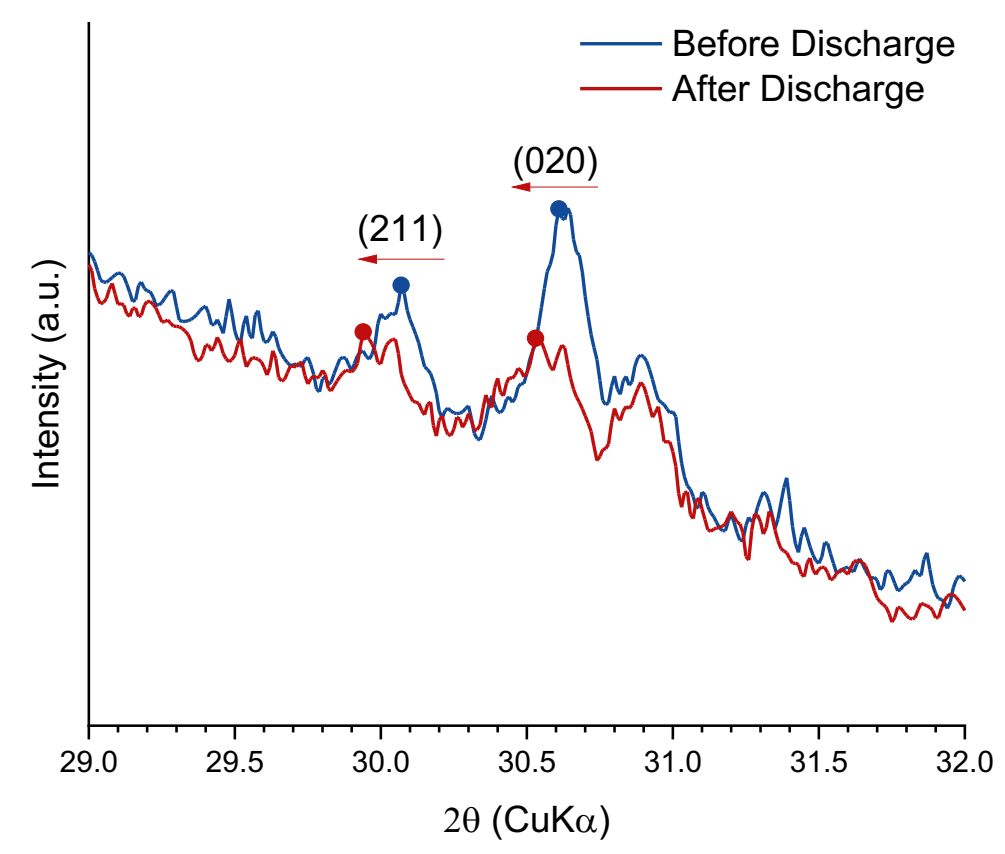

Supp. Figure 10: XRD spectra of electrode before and after the first discharge. Blue and red filled circles shown in the figure indicates the peak positions for the (211) and (020) planes before and after the potassium intercalation, respectively. 

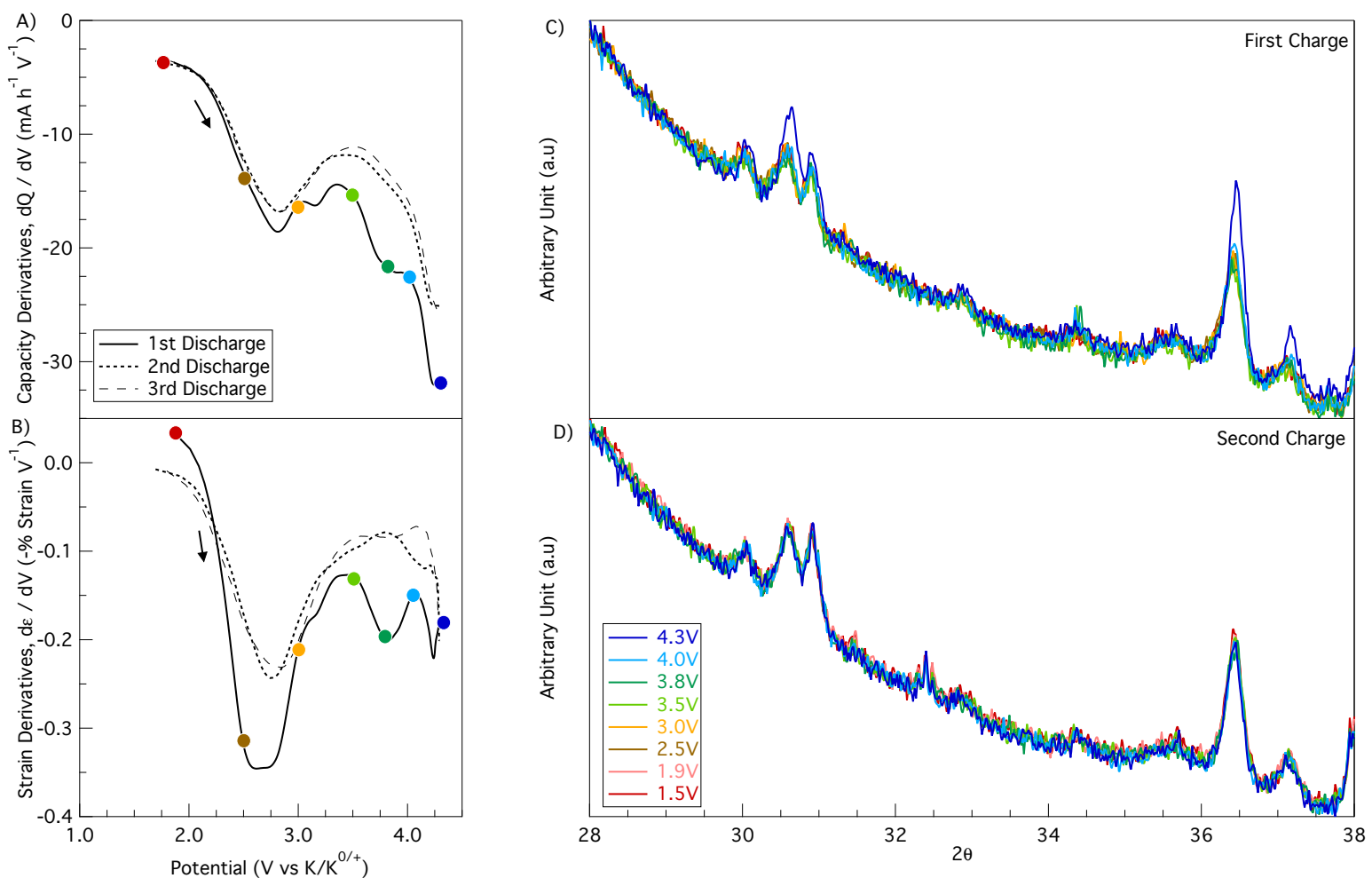

Supp. Figure 11: The figure is same as the Fig 5 in the manuscript. Only, the XRD patterns are plotted on top of each other in this figure. Structural, physical and electrochemical response of the $\mathrm{FePO}_{4}$ during charge cycles A) capacity and B) strain derivatives with respect to voltage. C-D) Corresponding $\mathrm{XRD}$ patterns at selected potentials colored as shown in the figure. 


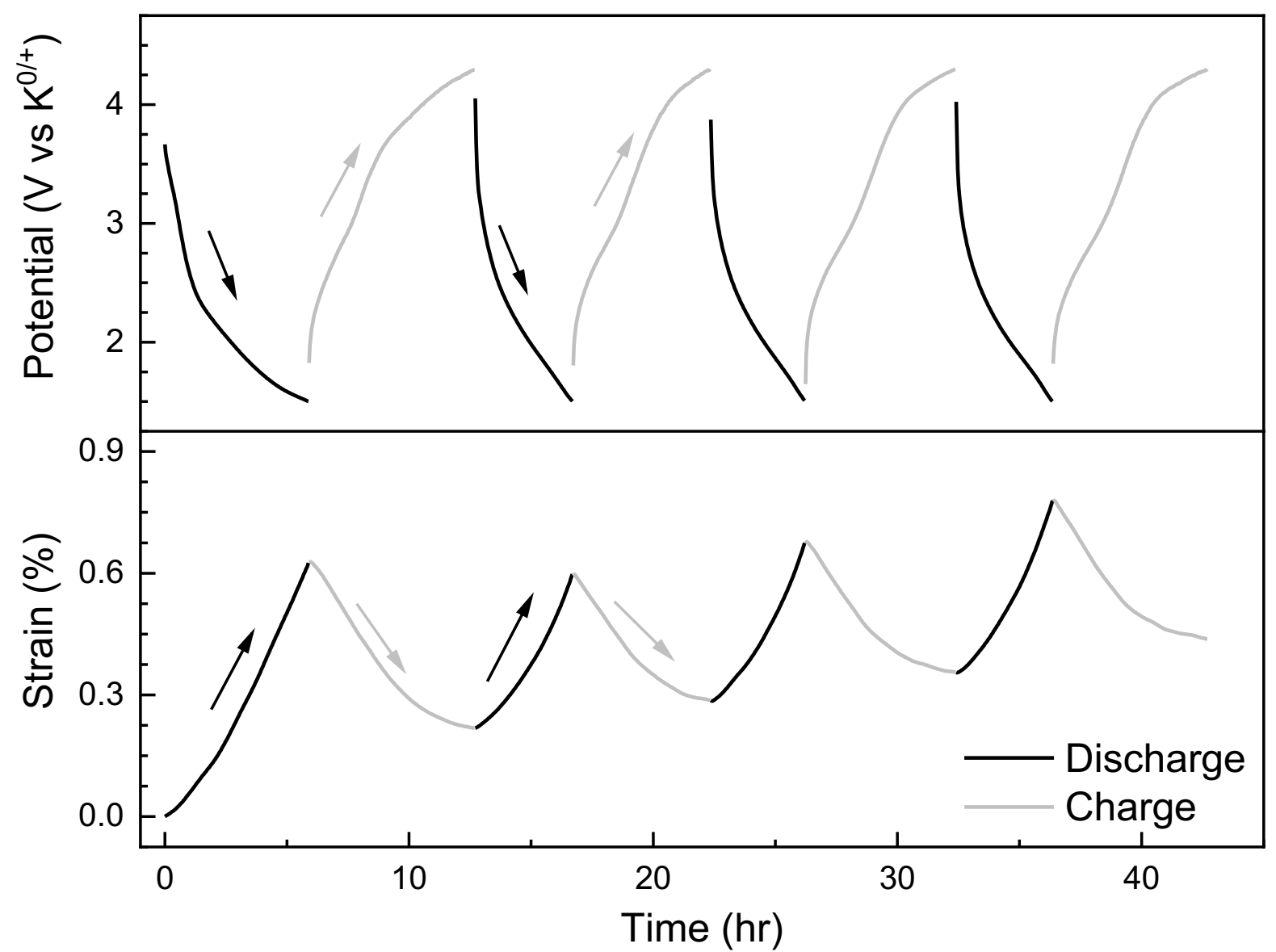

Supp. Figure 12: Voltage and strain evolution in iron phosphate electrode during potassium intercalation at $\mathrm{C} / 25$ rate in $0.5 \mathrm{M} \mathrm{KPF}_{6}$ in $\mathrm{PC}$ electrolyte 

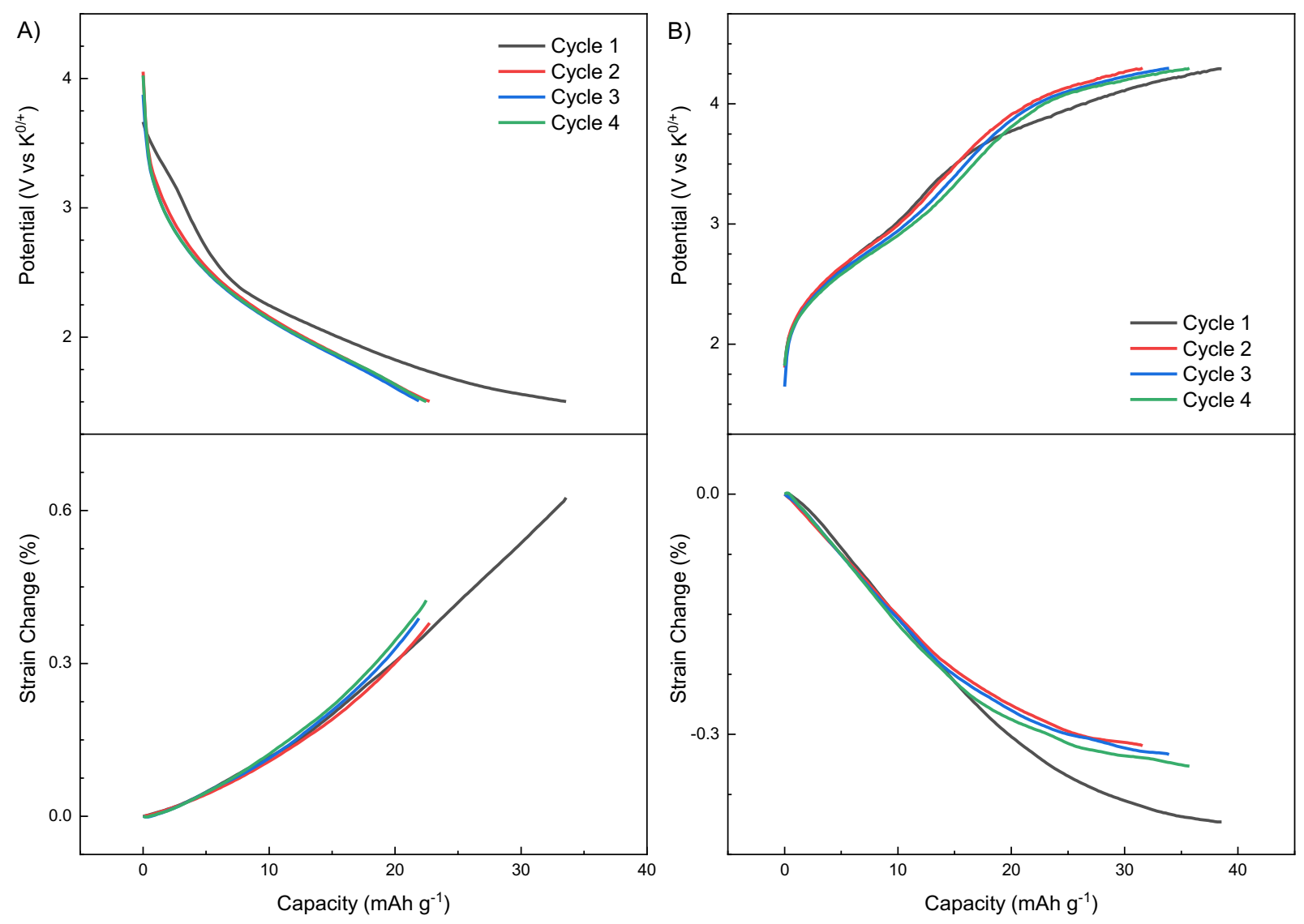

Supp. Figure 13: Change in strain versus (A) discharge and (B) charge capacity in iron phosphate during potassium intercalation at $\mathrm{C} / 25$ rate in $0.5 \mathrm{M} \mathrm{KPF}_{6}$ in $\mathrm{PC}$ electrolyte. Strain values are set to zero at the beginning of each charge and discharge cycles for better comparison. 

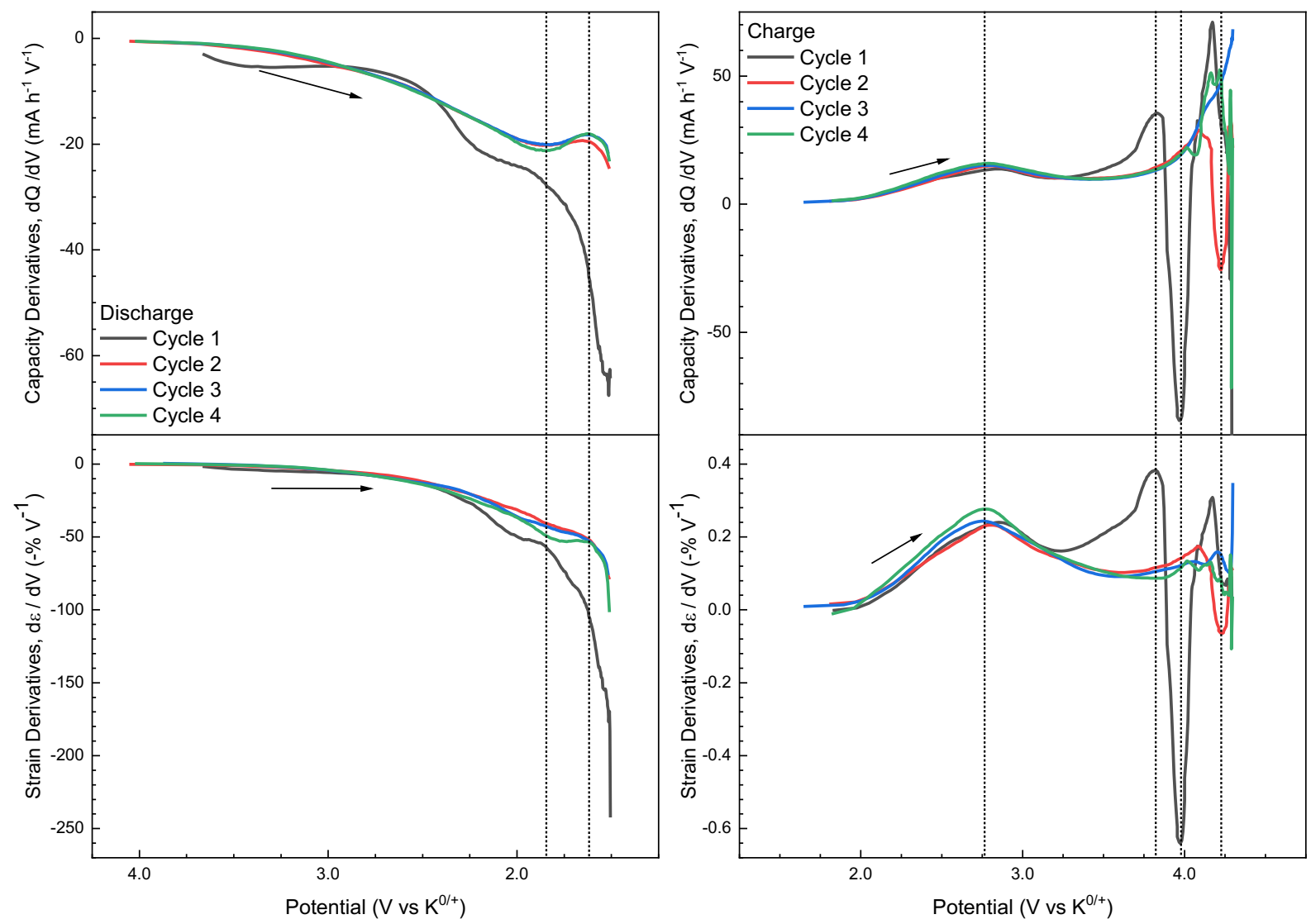

Supp. Figure 14: Capacity and strain derivatives in iron phosphate electrode during potassium intercalation for (A) discharge and (B) charge at $\mathrm{C} / 25$ rate in $0.5 \mathrm{M} \mathrm{KPF}_{6}$ in $\mathrm{PC}$ electrolyte 


\section{References}

1. Heubner, C., Heiden, S., Schneider, M. \& Michaelis, A. In-situ preparation and electrochemical characterization of submicron sized $\mathrm{NaFePO} 4$ cathode material for sodiumion batteries. Electrochim. Acta 233, 78-84 (2017).

2. Heubner, C., Heiden, S., Matthey, B., Schneider, M. \& Michaelis, A. Sodiation vs. Lithiation of FePO4: A comparative kinetic study. Electrochim. Acta 216, 412-419 (2016).

3. Çapraz, Rajput, S., White, S. \& Sottos, N. R. Strain Evolution in Lithium Manganese Oxide Electrodes. Exp. Mech. 58, 561-571 (2018).

4. Jones, E. M. C., Silberstein, M. N., White, S. R. \& Sottos, N. R. In Situ Measurements of Strains in Composite Battery Electrodes during Electrochemical Cycling. Exp. Mech. 54, 971-985 (2014). 